\title{
Poroelastic Center of Dilation Revisited
}

\author{
Alexandre Huynen ${ }^{\mathrm{a}}$
}

April, 2012

${ }^{a}$ Geomechanics, Department of Civil Engineering, University of Minnesota, MN, USA

\begin{abstract}
In both elasticity and poroelasticity, the nucleus of strain is usually used to refer to a singular solution such as the point force, the force dipole, the centers of rotation and dilation, etc. These fundamentals solutions constitute the building blocks for various problems of practical importance and are, therefore, relatively well understood. However, a comparison of the original elastic solution for the center of dilation with the classic Lamé solution reveals an unexpected dependence of the center of dilation solution on the Poisson's ratio. This inconsistency is investigated by means of two alternative representations of the singularity and leads to the identification of a compressed zone inside the singularity. The poroelastic equivalent to this nucleus of strain is shown to be the superposition of the elastic one with drained material parameters and an instantaneous point fluid source associated with a finite volume of fluid that is expelled from the compressed region inside the singularity and is injected in the porous medium.
\end{abstract}

\section{Introduction}

In elasticity, the classic solution obtained by Kelvin (1882) for a point force acting in a solid of infinite extent is the building block of what Boussinesq (1885) first called a nucleus of strain. Various nuclei of strain exist; all can be derived from Kelvin solution. For instance, the classic solutions for the double force with and without moment can be obtained by differentiation of the point force solution and, in turn, combined to form other nuclei such as the center of dilatation and the center of rotation. The solutions of these two particular nuclei of strain appear to have been first obtained by Dougall (1897) and were later quoted by Love (1920).

Interpreting the center of dilation as the insertion of an isotropic eigenstrain of strength $V$ (of dimension $\left[L^{3}\right]$ in space and $\left[L^{2}\right]$ in plane strain) at one point of an infinite elastic solid, Dougall solution can equivalently be build by superposition of dislocation dipoles. An examination of the resulting solution reveals that the displacement and stress fields vary in $1 / r^{2}$ and $1 / r^{3}$, respectively. This general behavior is in accordance with the classic Lamé solution (1852) and Love's intuition who stated that "The point must be in a cavity within the body [...]" (1920). However, while Dougall solution for the center of dilation depends on both shear modulus $G$ and Poisson ratio $\nu$, a solution derived from Lamé solution for a spherical cavity would result in a displacement field independent of Poisson's ratio, consistent with the zero volumetric strain associated with the $1 / r^{2}$ decay of the radial displacement. This unexpected dependence of Dougall solution on $\nu$ can equally be observed in plane strain.

Furthermore, it is well known that a certain parallelism exists between poroelasticity and elasticity; nuclei of strain are no exception. They are, therefore, build on the poroelastic analogue of Kelvin solution which has been derived by Cleary (1977) and later corrected by Rudnicki (1981, 1986). However, because of the inherent diffusive process that takes place in the porous medium, the nucleus of strain build by superposition of double forces exhibits a decaying strength and one must resort to dislocation dipoles to build a center of dilation with constant strength $V$. 
The corresponding solution is seen to be the superposition of an elastic center of dilation with a drained Poisson ratio (Dougall solution), and a point fluid source associated with a finite volume of fluid instantaneously injected in the porous medium $V_{f}=4 \eta V / 3$ (where $\eta$ is a poroelastic constant defined in the range 0 to 1/2). At large time, the poroelastic solution reduces to Dougall elastic solution only. This is again an unexpected behavior, if the nucleus of strain is viewed as a degenerated Lamé solution, which is the same in elasticity and in poroelasticity. Similar results are obtained for the plane strain center of dilation with, for this particular loading, $V_{f}=\eta V$.

The objectives of this paper are to (i) clarify the unexpected dependance of Dougall solution on Poisson ratio, and (ii) identify the origin of the fluid seemingly injected in the porous medium by the insertion of the center of dilation. The complexity concealed by the apparent simplicity this nucleus of strain was not the unique motivation that drove the authors. Indeed, the extensive use of the center of dilation in diverse fields even though its interpretation is unclear or debatable is remarkable; some of them are introduced in the following paragraphs.

The center of dilation and its opposite, the center of compression, have been widely used to describe lattice defects in crystals such as vacant sites or interstitial and impurity atoms (Eshelby, 1954, 1956; Kröner, 1959). Agglomeration of these points defect, e.g. precipitates, or more complex systems have been modeled by integration of the solution for the center of dilation over regions of various shapes; the resulting displacement and stress fields have been compared with those from the corresponding dislocation loops and slight differences have been revealed (Koehler, 1966; Groves and Bacon, 1969).

In volcanology, Anderson (1936) was the first to propose an analytical model to explain the formation or collapse of calderas. His representation of the magma chamber by a center of dilation was eventually followed by Mogi (1958) who concluded that geodetically measured elevation change and horizontal displacements associated with eruptions in Japan and Hawaii resulted from inflation and deflation of magma bodies within the volcanoes. What later became Mogi's model is now the most widely used method to predict the stresses and displacements generated by the pressure changes in a relatively deep-seated, spherical magma chamber (Gudmundsson, 1998; Lisowski, 2006). Relatively more complex distributions of centers of dilation have also been used to approximate various shapes of magmatic chambers (Segall, 2010).

In rock mechanics and in petroleum engineering, a similar idea initially introduced by McCann and Wilts (1951) has later been extended by Geertsma (1966, 1973a,b) to evaluate land subsidence provoked by fluid extraction or pressure changes in a subsurface reservoir. Distributions of centers of dilation have not only been used in the contexts of hydrocarbon production and storage but have been applied, with the same relevance, to aquifer management and carbon sequestration. More recently, Segall (1989, 1992); Segall et al. (1994) evaluated the effects of fluid extraction on the occurrence of seismicity using similar distributions of centers of dilation.

\section{Center of Dilation}

To answer these questions, both elastic and poroelastic solutions for the center of dilation are first closely examined. The convention for the superscripts used to designate the various singularities on which this nucleus of strain is build is given in Table 1 .

\section{$2.1 \quad$ Elasticity}

As it has already been emphasized in the introduction, the center of dilation is a classic nucleus of strain that can be obtained from Kelvin (1882) fundamental solution by differentiation and superposition. It is, however, recognized that singular solutions can equivalently be obtained by integration of Navier elastic equation in which proper Dirac delta functions have been inserted. For instance, the introduction of the singularity $F_{i}=\delta_{i k} \delta(\boldsymbol{x})$ for the body force in Navier equations generates Kelvin solution for a point force acting in the $x_{k}$-direction. According to the nomenclature introduced in Table 1 , the corresponding displacement field is referred to as $u_{i k}^{F}$. 
Table 1: Convention for naming singular solutions

\begin{tabular}{lccc}
\hline Singularity type & Elastic & Instantaneous & Continuous \\
Fluid source $(\gamma)$ & - & $s i$ & $s c$ \\
Fluid dipole $\left(\gamma_{i}\right)$ & - & $p i$ & $p c$ \\
Ring fluid source & - & $a i$ & $a c$ \\
Ring fluid dipole & - & $b i$ & $b c$ \\
Total force $\left(F_{i}\right)$ & $F$ & $F i$ & $F c$ \\
Displacement discontinuity $\left(E_{i j}\right)$ & $d$ & $d i$ & $d c$ \\
Edge dislocation & $e$ & $e i$ & $e c$ \\
Ring dislocation & $r$ & $r i$ & $r c$ \\
Center of dilation & $c$ & $c i$ & $c c$ \\
\hline
\end{tabular}

Following Cheng and Detournay (1998) developments, one can also define the singularity $E_{i j}$ for the displacement discontinuity such that its introduction in Navier equations leads to

$$
G u_{i, j j}+\frac{G}{1-2 \nu} u_{j, j i}=-F_{i}+2 G E_{i j, j}+2 G \frac{\nu}{1-2 \nu} E_{j j, i}
$$

with the displacement discontinuity fourth-order tensor defined as

$$
E_{i j k l}=-\frac{1}{2}\left(\delta_{i k} \delta_{j l}+\delta_{j k} \delta_{i l}\right) \delta(\boldsymbol{x})
$$

where $\delta_{i j}$ is the Kronecker delta and $\delta(\cdot)$ the Dirac delta function. Combining these two equations and identifying the derivative of a delta function $\delta_{i k} \delta_{, l}(\boldsymbol{x})$ with a double force $u_{i k, l}^{F}$, it can be shown that

$$
u_{i k l}^{d}=G\left(u_{i k, l}^{F}+u_{i l, k}^{F}\right)+2 G \frac{\nu}{1-2 \nu} \delta_{k l} u_{i m, m}^{F}
$$

where $u_{i k l}^{d}$ is the solution for the dislocation dipole. This relation, which is exactly the constitutive relation of an isotropic elastic solid, expresses the link that exists between dislocation dipoles and double forces (Cheng and Detournay, 1998).

From Eq. (3), it also appears that Dougall original solution for the 3D center of dilation is equivalent to the superposition of three mutually orthogonal dislocation dipoles, each producing a normal opening of magnitude $V / 3$. In the particular configuration of plane strain loading, the center of dilation is obtained by superposition of two mutually orthogonal dislocation dipoles of magnitude $V / 2$ such that the total strength of the singularity is again $V$. The resulting displacement fields

$$
\begin{aligned}
u_{i}^{c} & =\frac{V(1+\nu)}{12 \pi(1-\nu)} \frac{r_{, i}}{r^{2}} & & \text { for } 3 \mathrm{D} \\
& =\frac{V}{4 \pi(1-\nu)} \frac{r_{, i}}{r} & & \text { for } 2 \mathrm{D}
\end{aligned}
$$

clearly exhibit the former mentioned dependence on Poisson ratio.

As emphasized in the introduction, alternative singularities associated with the same decay of the displacement field can be derived from the classic Lamé solution (1852) for spherical and cylindrical cavities. One can, indeed, imagine the limiting process consisting in the application of a radial displacement of magnitude $V / 4 \pi a^{2}$ (resp. $V / 2 \pi a$ ) at the boundary of a spherical (resp. cylindrical) cavity of radius $a$, with $a$ tending to zero. Consistently with the zero volumetric strain associated with 
these solutions, the resulting displacement fields

$$
\begin{aligned}
u_{i} & =\frac{V}{4 \pi} \frac{r_{, i}}{r^{2}} & & \text { for } 3 \mathrm{D} \\
& =\frac{V}{2 \pi} \frac{r_{, i}}{r} & & \text { for } 2 \mathrm{D}
\end{aligned}
$$

are independent of any material parameters.

\subsection{Poroelasticity}

The linear, isotropic, theory of poroelasticity, as originally proposed by Biot (1941), relies on a conceptual model consisting of a pore fluid moving freely in a coherent solid skeleton and three key assumptions: (i) linearity between stresses and strain, (ii) reversibility under small strain (meaning that no energy is dissipated during a closed loading cycle), and (iii) isotropy and homogeneity of the porous solid. Adopting a stress-strain formulation similar to the one initially introduced by Biot, the total stress $\sigma_{i j}$ and the pore pressure $p$ are chosen to be the basic dynamical variables of the constitutive equations. A positive normal stress implies tension while a positive pore pressure is treated as compressive. The corresponding kinematical variables are the solid strain $\varepsilon_{i j}$ and the variation of fluid content $\zeta$, defined as the variation of fluid volume per unit volume of porous solid.

As shown by Detournay and Cheng (1993) for instance, the equations governing this linear, isotropic, theory of poroelasticity can be combined to obtain a set of four field equations expressed in terms of the solid displacement $u_{i}$ and the variation of fluid content $\zeta$. Doing so, a poroelastic version of Navier equations is derived

$$
G u_{i, j j}+\frac{G}{1-2 \nu_{u}} u_{j, j i}=-F_{i}+2 G E_{i j, j}+2 G \frac{\nu}{1-2 \nu} E_{j j, i}+\frac{G\left(\nu_{u}-\nu\right)}{\eta(1-\nu)\left(1-2 \nu_{u}\right)} \zeta_{, i}
$$

where $F_{i}$ is the body force per unit volume of the bulk material and $\eta$ is the poroelastic stress coefficient defined as

$$
\eta=\frac{\alpha(1-2 \nu)}{2(1-\nu)}
$$

with $\nu_{u}$ the undrained Poisson ratio and $\alpha$ the Biot stress coefficient. The coupling term, which acts as an additional body force, is governed by the following diffusion equation

$$
\frac{\partial \zeta}{\partial t}-c \nabla^{2} \zeta=\frac{\eta c}{G} F_{i, i}+\gamma-\kappa f_{i, i}-2 \eta c\left(E_{i j, j i}-\nabla^{2} E_{j j}\right)
$$

where $c$ is the diffusivity and $\gamma$ is the fluid source density.

In the particular configuration of infinite domain, a certain uncoupling of these equations can be achieved. This uncoupling allows to express the solution as the sum of two components: (i) an undrained one, $u_{i}^{0}$, which is time-independent and corresponds to the instantaneous displacement field induced at $t=0^{+}$, and (ii) an irrotational component, $\Delta u_{i}$, which incorporates the diffusion process. At the instant of loading, the poroelastic medium behaves as an elastic one with undrained material parameters and the instantaneous response $u_{i}^{0}$ is therefore entirely governed by Navier equations with undrained material parameters. This particularly useful result enables one to deduce the instantaneous solution for poroelastic nuclei of strain from their elastic homologue by substitution of the undrained Poisson ratio $\nu_{u}$ for the drained one. On the other hand, as $t \rightarrow \infty$, the diffusion process dissipates and the classic elastic solution is recovered.

Following the reasoning exposed in the previous section, one can build the continuous poroelastic center of dilation by superposition of dislocation dipoles. Using either the solution of Carvalho and Curran (1998) or Cheng and Detournay (1998) for the continuous dislocation dipole ${ }^{1}$ (see Appendix A.1), the solution is seen to be the superposition of the elastic center of dilation Eq. (4) and

\footnotetext{
${ }^{1}$ The plane strain solution for the dislocation dipole has independently been obtained by Curran and Carvalho (1987) and Detournay and Cheng (1987).
} 
an instantaneous point fluid source

$$
u_{i}^{c c}=u_{i}^{c}+V_{f} u_{i}^{s i}
$$

Similarly, the variation of fluid content resulting from the insertion of the center of dilation is given by

$$
\zeta^{c c}=V_{f} \zeta^{s i}
$$

where the volume of fluid that appears to be instantaneously injected in the porous medium is $V_{f}=$ $4 \eta V / 3$ for the general $3 \mathrm{D}$ loading and $V_{f}=\eta V$ for plane strain loading. This result, that leads to an apparent increase of the total fluid content inside the porous medium, is relatively surprising since no fluid has actually been injected in the medium. At large time, the excess of pore pressure induced by the fluid source dissipates and the displacement field reduces to the elastic center of dilation given by Eq. (4) or (5) depending on the nature of the loading.

\section{Exploded View of the Singularity}

To understand the mechanism behind the poroelastic center of dilation and reveal where the seemingly injected fluid comes from, we look "inside" the singularity and consider an exploded view of it. The physics of the problem is first investigated under the assumption of plane strain loading, and then extended to the more general 3D center of dilation.

\subsection{Plane Strain Solution}

In plane strain, the displacement discontinuity dyad shown in Figure 1 is substituted for the center of dilation and the two dislocation dipoles are seen as the limit of two orthogonal dislocation segments of length $2 a$ and displacement discontinuity $D_{n}=V / 4 a$ (with $D_{n}>0$ for an opening discontinuity) such that the volume instantaneously created by the singularity is $V$. Both displacement discontinuity segments forming the DD-dyad are created by superposition of two edge dislocations and the solution therefore results from the superposition of four edge dislocations

$$
u_{i}=\frac{V}{4 a}\left[u_{i 1}^{e c}\left(x_{1}+a, x_{2}\right)-u_{i 1}^{e c}\left(x_{1}-a, x_{2}\right)+u_{i 2}^{e c}\left(x_{1}, x_{2}+a\right)-u_{i 2}^{e c}\left(x_{1}, x_{2}-a\right)\right]
$$

where $u_{i j}^{e c}$ is the displacement field due to a continuous edge dislocation aligned with $x_{j}$-axis. For brevity, we introduce the operator $\mathcal{D}_{k}^{a}$ such that the previous expression can be rewritten as

$$
u_{i}=\frac{V}{2} \mathcal{D}_{k}^{a} u_{i k}^{e c}
$$

with the particularity that

$$
\lim _{a \rightarrow 0} \mathcal{D}_{k}^{a}=\partial_{k}
$$

where $\partial_{k}$ is the partial derivative with respect to $x_{k}$.

Form this definition, it can easily be shown that the solution for the center of dilation is recovered by shrinking the DD-dyad, i.e. passing to the limit for $a$ tending to 0 ,

$$
\begin{aligned}
u_{i}^{c c} & =\frac{V}{2} \lim _{a \rightarrow 0} \mathcal{D}_{k}^{a} u_{i k}^{e c} \\
& =\frac{V}{2} u_{i k, k}^{e c} \\
& =\frac{V}{2} u_{i k k}^{d c}
\end{aligned}
$$

where the relation $u_{i k, k}^{e c}=u_{i k k}^{d c}$ has been used (Detournay and Cheng, 1987).

The definition of the exploded singularity presented in Figure 1 reveals that the solution manifests a 4 -fold rotational symmetry; meaning that any quantity remains unchanged by a rotation of $\pi / 2$ 


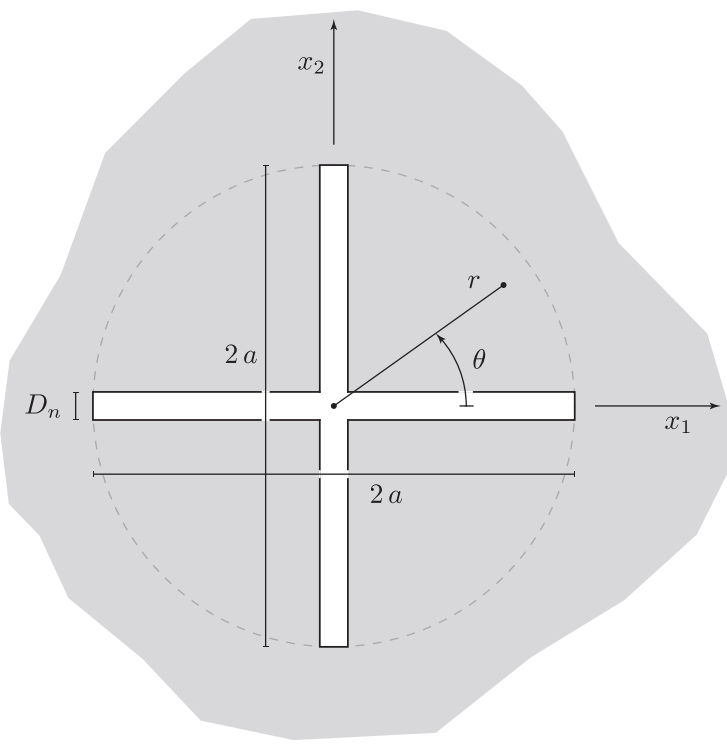

Figure 1: Definition of the displacement discontinuity dyad

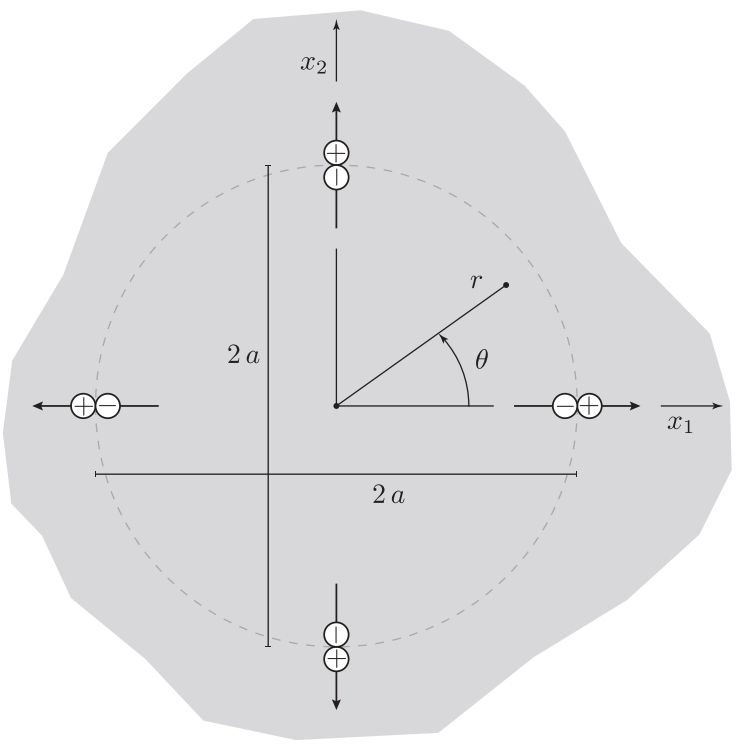

Figure 2: The two pairs of equal and opposite dipoles

radian. This definition also reveals that the circle circumscribed to the displacement discontinuity dyad divides the domain in two regions: (i) an inner one, corresponding to the disc of radius $a$ and which is not actually visible in the original representation of the centre of dilation, and (ii) an outer one, consisting of the infinite porous medium complementary to the disk. Finally, two characteristic parameters are naturally identified: the length scale $r^{*}=a$ and the time scale $t^{*}=a^{2} / 4 c$.

As already emphasized, the solution (9) can be decomposed in two components: (i) an undrained one, $u_{i}^{0}$, which is time-independent and corresponds to the initial displacement induced at time $t=0^{+}$, and (ii) a time-dependent component, $\Delta u_{i}$, which incorporates the diffusion process.

\subsubsection{Instantaneous Response}

At the instant of loading, the poroelastic medium behaves as an elastic one with undrained material parameters and the instantaneous response $u_{i}^{0}$ is therefore entirely governed by Navier equation with undrained material parameters. Adopting a notation similar to the one introduced previously, yields

$$
u_{i}^{0}=\frac{V}{2} \mathcal{D}_{k}^{a} u_{i k}^{e c, 0}
$$

where $u_{i k}^{e c, 0}$ is the classic elastic solution for an edge dislocation (Dundurs, 1969) with undrained Poisson's ratio. Both radial and angular components of the instantaneous displacement field are presented in Figure 3. This solution, being the superposition of four singularities, is not conveniently manipulable and will not be explicitly given here. A concise expression for the instantaneous volumetric strain $\epsilon^{0}$ can, however, be obtained by taking advantage of the 4 -fold rotational symmetry and switching to polar coordinates

$$
\epsilon^{0}=-\frac{V\left(1-2 \nu_{u}\right)}{2 \pi a^{2}\left(1-\nu_{u}\right)} \frac{1-\rho^{4} \cos (4 \theta)}{1+\rho^{8}-2 \rho^{4} \cos (4 \theta)}
$$

where $\rho=r / a$ and $\theta$ is the polar angle, which has a branch cut along the positive $x_{1}$-axis $(0 \leq \theta \leq 2 \pi)$, see Figure 1. The instantaneous volumetric strain $\epsilon^{0}$ is presented in Figure 4, the light blue region lying between the $\epsilon^{0}=0$ level curves corresponds to compression while the darker region lying outside these curves correspond to dilation of the porous solid. 


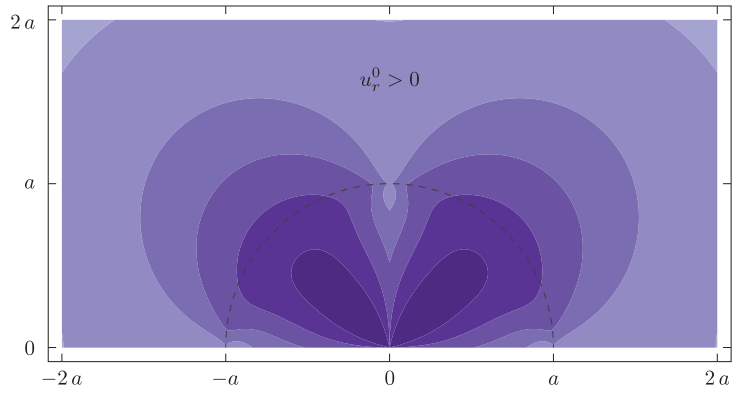

(a) Radial displacement $u_{r}^{0}$, inner region

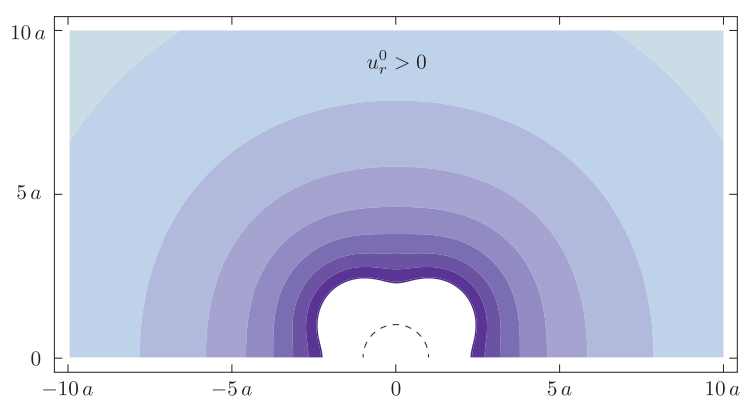

(c) Radial displacement $u_{r}^{0}$, outer region

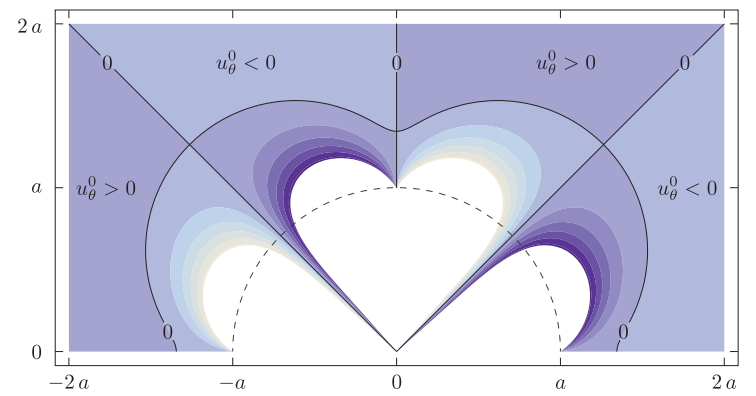

(b) Angular displacement $u_{\theta}^{0}$, inner region

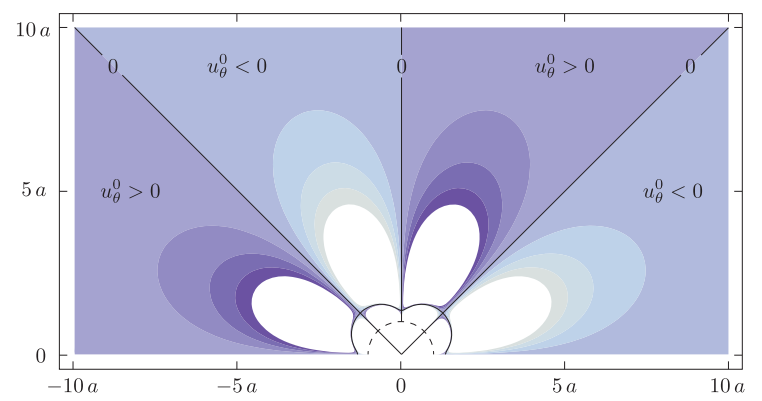

(d) Angular displacement $u_{\theta}^{0}$, outer region

Figure 3: Instantaneous displacement field

It can be shown, by integration of the previous expression, that a volume of solid $\Delta V_{c}^{0}$ is instantaneously compressed in the inner region due to the insertion of the DD-dyad

$$
\begin{aligned}
\int_{0}^{a} \int_{0}^{2 \pi} \epsilon^{0} r \mathrm{~d} \theta \mathrm{d} r & =-\frac{1-2 \nu_{u}}{2\left(1-\nu_{u}\right)} V \\
& =-\Delta V_{c}^{0}
\end{aligned}
$$

On the contrary and as expected from the symmetry of Figure 4, the outer region is characterized by a null mean volumetric strain $\left\langle\epsilon^{0}\right\rangle$

$$
\begin{aligned}
\left\langle\epsilon^{0}\right\rangle & =\frac{1}{2 \pi r} \int_{0}^{2 \pi} \epsilon^{0} r \mathrm{~d} \theta \\
& =-\frac{\Delta V_{c}^{0}}{\pi a^{2}}(1+\operatorname{sign}(a-r))
\end{aligned}
$$

The 4-fold rotational symmetry of the solution is emphasized by writing the asymptotic expansion of the displacement field (11). In the outer region, i.e. for $\rho \gg 1$, we have

$$
\begin{aligned}
& u_{r}^{0}=\frac{V}{4 \pi\left(1-\nu_{u}\right) r}+\frac{V}{8 \pi a} \sum_{k=1}^{n-1} \frac{\mathcal{P}_{k}^{r}}{\rho^{4 k+1}} \cos (4 k \theta)+\mathcal{O}\left(\rho^{-4 n+1}\right) \\
& u_{\theta}^{0}=\frac{V}{8 \pi a} \sum_{k=1}^{n-1} \frac{\mathcal{P}_{k}^{\theta}}{\rho^{4 k+1}} \cos (4 k \theta)+\mathcal{O}\left(\rho^{-4 n+1}\right)
\end{aligned}
$$

where the $\mathcal{P}_{k}^{r}$ and $\mathcal{P}_{k}^{\theta}$ are binomials of $\rho$. When the DD-dyad is shrunk to a center of dilation, or equivalently at large distance of it, only the first term of Eq. (13) remains and the classic elastic 


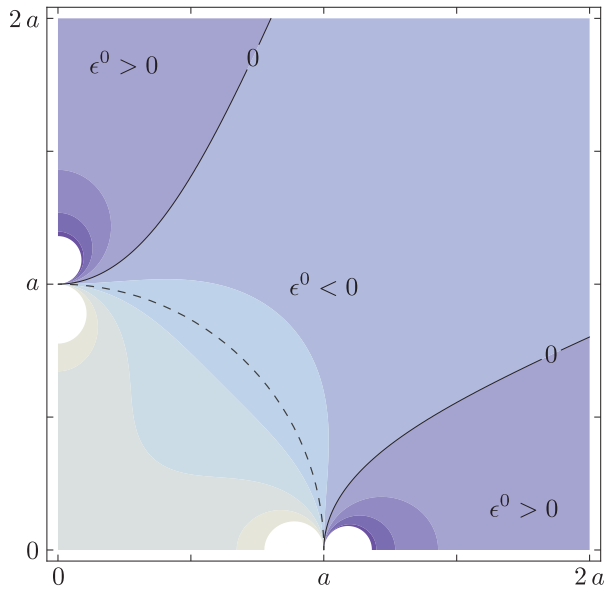

(a) In the inner region

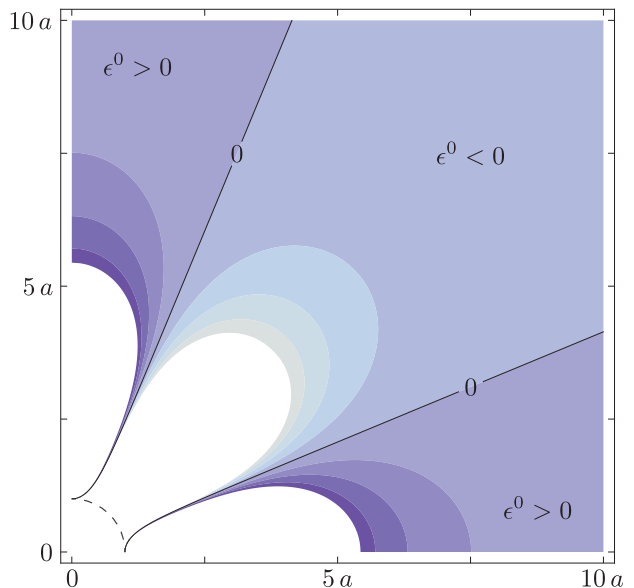

(b) In the outer region

Figure 4: Instantaneous volumetric strain $\epsilon^{0}=\varepsilon_{r}^{0}+\varepsilon_{\theta}^{0}$. The solid is compressed inside the $\epsilon^{0}=0$ level curve (light blue), and is dilated outside.

solution with undrained material parameters is recovered. Expressions similar to Eqs. (13) and (14) can be obtained for the inner region, i.e. for $\rho \ll 1$.

The volume of solid instantaneously displaced in the outer region $\Delta V_{s}^{0}=V-\Delta V_{c}^{0}$ can equivalently be obtained by integration of the radial displacement over a circle of radius $r$. Using the asymptotic expansion (13) for $u_{r}^{0}$ and expanding the sum to infinity, one can show that this volume is independent of the radius at which it is observed (for $r \geq a$ )

$$
\begin{aligned}
\Delta V_{s}^{0} & =\int_{0}^{2 \pi} u_{r}^{0} r \mathrm{~d} \theta \\
& =\frac{V}{2\left(1-\nu_{u}\right)}
\end{aligned}
$$

Actually, it appears that only the first term of the expansion, which also corresponds to the classic elastic solution with undrained Poisson's ratio, contributes to this integral.

These results express that only a fraction of the singularity strength is perceived in the outer region and that a finite volume of porous solid is instantaneously compressed in the inner region. Since these results are independent of $a$, they remain valid when the DD-dyad is shrunk to the center of dilation and, therefore, explain the appearance of Poisson ratio in the classic Dougall solution. Furthermore, because the pore pressure is allowed to return to its initial state, the compression of the inner region naturally results in the expulsion of interstitial fluid which, by continuity, is injected in the outer region. This diffusive precess, taking place for $t>0^{+}$, is investigated in the next section.

\subsubsection{Transient Response}

The instantaneous compression of the porous solid due to the insertion of the DD-dyad generates a nonuniform increase of pore pressure and, therefore, leads to an interstitial flow. This diffusion process is fully incorporated in the irrotational component $\Delta u_{i}$ of the complete solution (9). According to Detournay and Cheng (1987), the time-dependent part of the poroelastic edge dislocation is created by the spatial derivative of a fluid source, also known as fluid dipole. Consequently, the transient part of the solution for the DD-dyad results from the superposition of two pairs of equal and opposite fluid dipoles, each characterized by an intensity equal to $2 \eta c D_{n}$ and located at the tip of the DD-dyad, 
see Figure 2. The displacement field can, therefore, be expressed as

$$
\begin{aligned}
u_{i} & =u_{i}^{0}+\Delta u_{i} \\
& =u_{i}^{0}+\eta c V \mathcal{D}_{k}^{a} u_{i k}^{p c}
\end{aligned}
$$

where $u_{i}^{0}$ is the instantaneous part of the displacement, c.f. Eq. (11), and $u_{i k}^{p c}$ is the displacement due to a continuous fluid dipole aligned with the $x_{k}$-axis, see Appendix A.3.

Again, both the volume of solid displaced in the outer region and the volume of solid compressed in the inner one can be evaluated. Because the undrained material is stiffer than the drained one, the compressed volume $\Delta V_{c}$ progressively increases while, simultaneously, the displaced volume $\Delta V_{s}$ decreases in the same proportion such that $V=\Delta V_{c}+\Delta V_{s}$ remains constant; eventually leading to

$$
\begin{aligned}
\Delta V_{c}^{\infty} & =\frac{1-2 \nu}{2(1-\nu)} V \\
\Delta V_{s}^{\infty} & =\frac{V}{2(1-\nu)}
\end{aligned}
$$

at large time, i.e. for $t \rightarrow \infty$.

The variation of fluid content in the porous medium is given by

$$
\zeta=\eta c V \mathcal{D}_{k}^{a} \zeta_{k}^{p c}
$$

and presented in Figure 5 for various values of the dimensionless time $\tau=t / t^{*}$. Interstitial fluid is expelled from the light blue regions lying between the $\zeta=0$ level curves, and absorbed by the darker regions lying outside these curves. As the dimensionless time $\tau$ increases, the region corresponding to negative variation of fluid content grows progressively and, in time, covers entirely the regions that were instantaneously compressed by the insertion of the DD-dyad, see Figure 4.

As seen in Figure 5, interstitial fluid is also gradually expelled from the inner region and, by continuity, injected in the outer one. The corresponding volume of fluid $V_{f}$, which can be be obtained by integration of $\zeta$ over the disc of radius $a$, and the volume of solid $\Delta V_{s}$ are presented in Figure 6 as functions of $\tau=t / t^{*}$. Beyond the fact that they exhibit a very similar time-dependency, we note that the volume of fluid $V_{f}$ is bounded. Indeed, since the pore pressure is allowed to diffuse and regain its initial state, the fluid volume gained (or lost) in a material element is related to the volume change of that element by the relation $\zeta^{\infty}=\alpha \epsilon^{\infty}$ for $t \rightarrow \infty$. Therefore, we have

$$
\begin{aligned}
\lim _{t \rightarrow \infty} V_{f} & =-\int_{0}^{2 \pi} \int_{0}^{a} \zeta^{\infty} r \mathrm{~d} r \mathrm{~d} \theta \\
& =-\alpha \int_{0}^{2 \pi} \int_{0}^{a} \epsilon^{\infty} r \mathrm{~d} r \mathrm{~d} \theta \\
& =\alpha \Delta V_{c}^{\infty}
\end{aligned}
$$

which can also be written as $V_{f}^{\infty}=\eta V$ by definition of the poroelastic stress coefficient $\eta$, see Eq. (7).

When the DD-dyad is shrunk to the center of dilation, the characteristic time $t^{*}=a^{2} / 4 c$ goes to zero and this volume of fluid appears to be instantaneously injected in the outer region. Mathematically, this shrinking operation, obtained by taking the length $a$ to zero, is equivalent to the application of the Laplace operator to a continuous point fluid source. Indeed, considering for instance the variation of fluid content given by Eq. (15), yields

$$
\begin{aligned}
\zeta^{c c} & =\eta c V \lim _{a \rightarrow 0} \mathcal{D}_{k}^{a} \zeta_{k}^{p c} \\
& =\eta c V \zeta_{, k k}^{s c}
\end{aligned}
$$

since a fluid dipole is the spatial derivative of a fluid source, i.e. $\zeta_{k}^{p c}=\zeta_{, k}^{s c}$. Additionally, the variation of fluid content must satisfies the diffusions equation $\zeta_{, t}^{s c}-c \zeta_{, k k}^{s c}=\gamma$ with $\gamma=\delta(\boldsymbol{x}) \mathrm{H}(t)$ for a continuous 


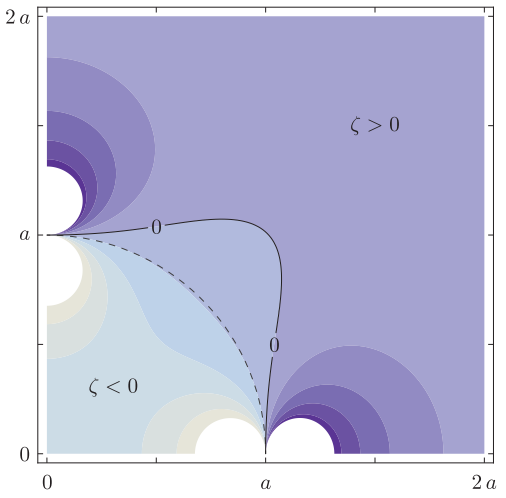

(a) $\tau=1$

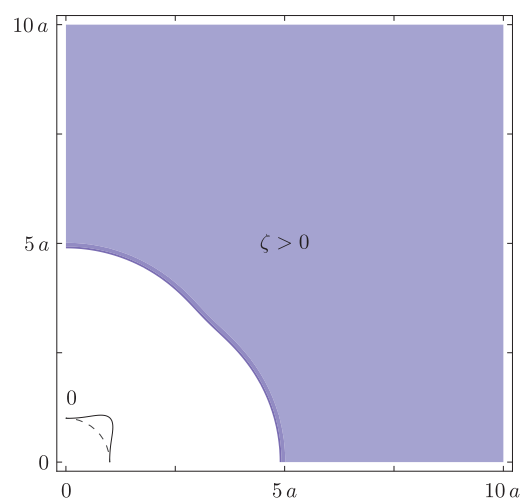

(d) $\tau=1$

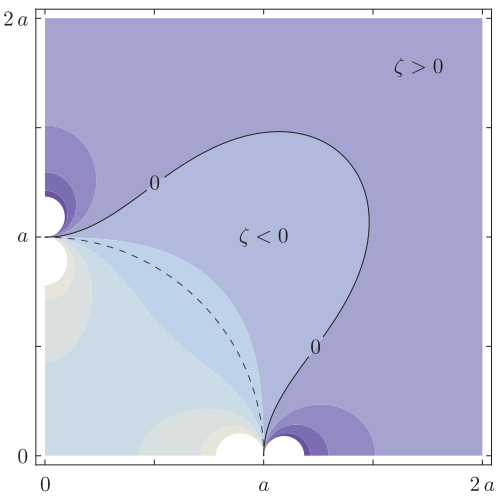

(b) $\tau=10$

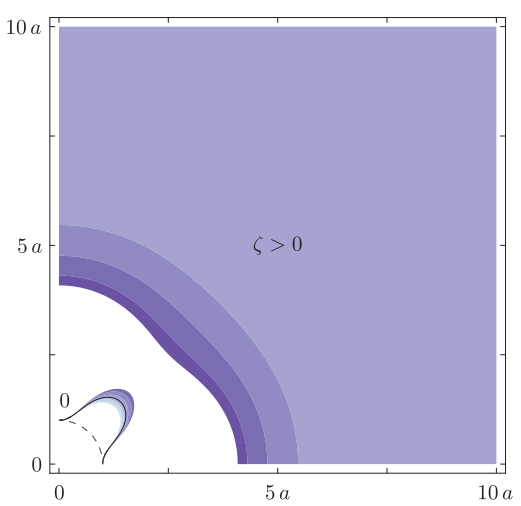

(e) $\tau=10$

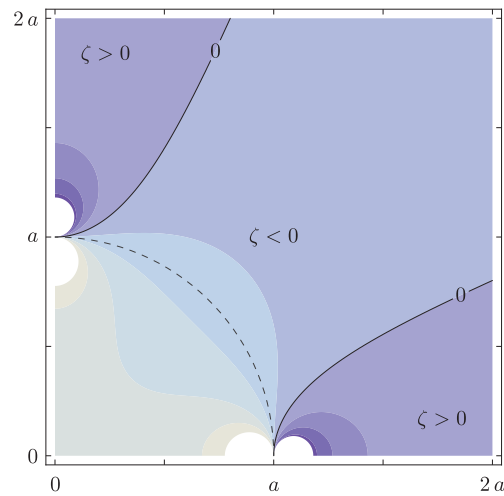

(c) $\tau \rightarrow \infty$

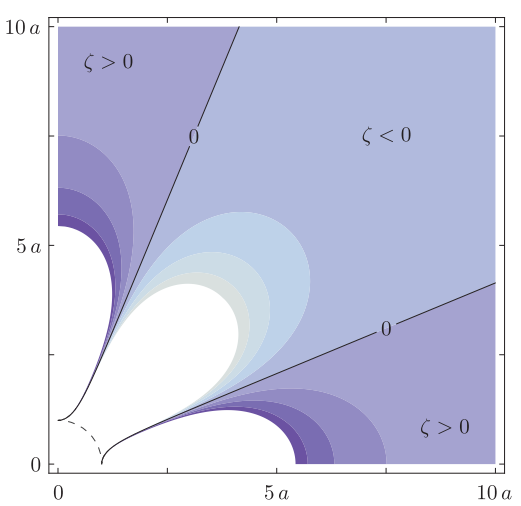

(f) $\tau \rightarrow \infty$

Figure 5: Variation of fluid content $\zeta$ in the inner and outer regions of the porous medium; interstitial fluid is expelled from the light gray regions lying between the $\zeta=0$ level curves, and absorbed by the darker regions lying outside these curves

point fluid source. Considering, moreover, that an instantaneous forcing function is the time derivative of a continuous one (Cheng and Detournay, 1998), the former relation reduces to

$$
\zeta^{c c}=V_{f}\left(\zeta^{s i}-\gamma\right)
$$

which expresses that the fluid $V_{f}=\eta V$ thought to be injected in the medium is actually withdrawn from the point at which the center of dilation is introduced, thus preserving the volume of fluid present in the porous medium.

\subsection{Three-dimensional Solution}

In $3 \mathrm{D}$, the ring dislocation triad shown in Figure 7 is substituted for the center of dilation and the three dislocation dipoles are seen as the limit of three, mutually orthogonal, continuous ring dislocations of diameter $2 a$ and displacement discontinuity $D_{n}=V / 3 \pi a^{2}$ (with $D_{n}>0$ for an opening discontinuity) such that the volume instantaneously created by the singularity is $V$. The corresponding solution can, therefore, be written as

$$
u_{i}=\frac{V}{3 \pi a^{2}}\left(u_{i 1}^{r c}+u_{i 2}^{r c}+u_{i 3}^{r c}\right)
$$

where $u_{i k}^{r c}$ is the displacement field due to a continuous ring dislocation of radius $a$ characterized by a Burger vector parallel to the $x_{k}$-axis. Again, the solution for the center of dilation can be recovered 


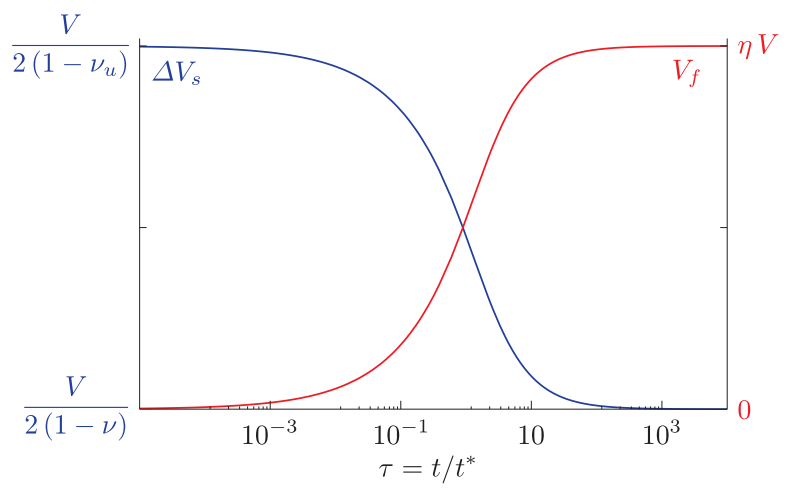

Figure 6: Volume of solid displaced by the DD-dyad in the outer region, $V_{s}$, and total volume of fluid expelled from the inner region, $V_{f}$

from this definition by shrinking the RD-triad, i.e. tending the length $a$ to zero. It can indeed be shown that the following relation holds

$$
\begin{aligned}
u_{i}^{c c} & =\frac{V}{3} \lim _{a \rightarrow 0} \frac{1}{\pi a^{2}}\left(u_{i 1}^{r c}+u_{i 2}^{r c}+u_{i 3}^{r c}\right) \\
& =\frac{V}{3}\left(u_{i 11}^{d c}+u_{i 22}^{d c}+u_{i 33}^{d c}\right)
\end{aligned}
$$

by considering that the solution for a ring dislocation can be obtained by integration of a normal displacement discontinuity over the disk circumscribed to this circle, see Appendix B.

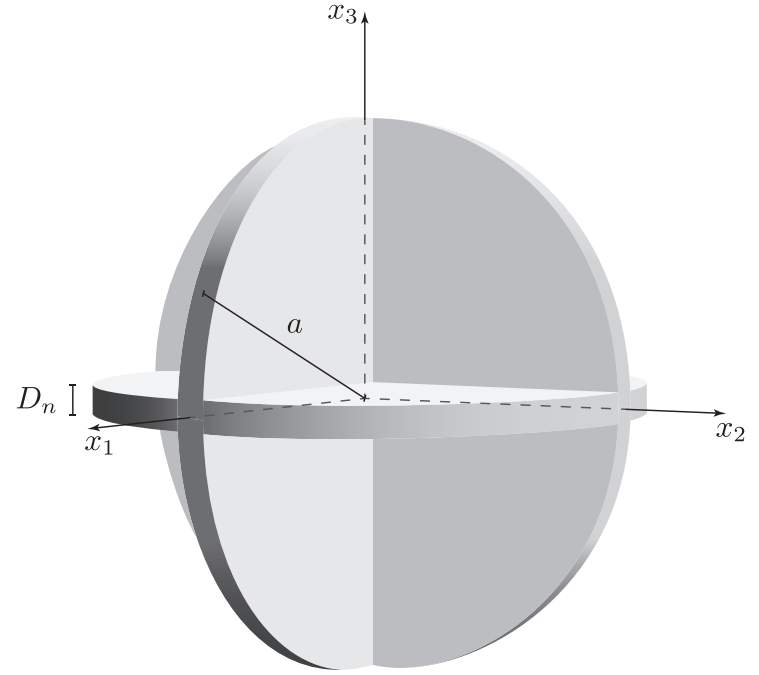

Figure 7: Definition of the ring dislocation triad

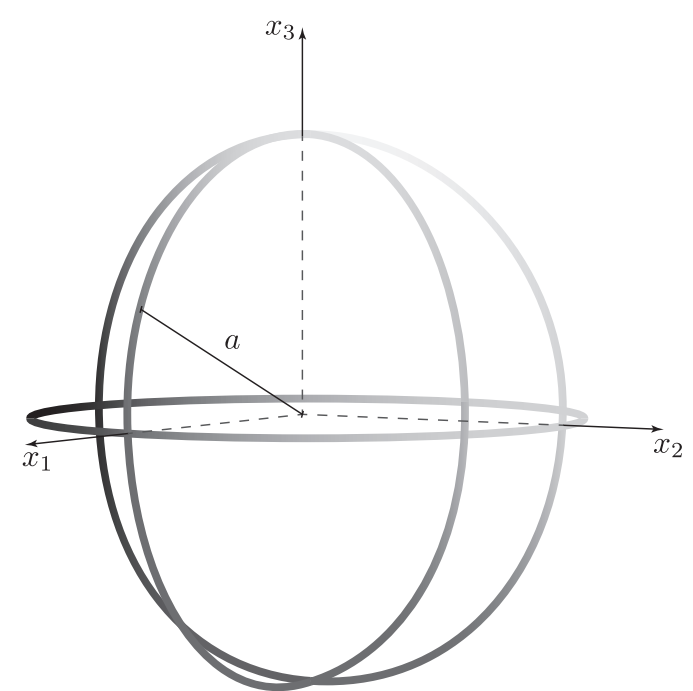

Figure 8: The three rings fluid dipole

By analogy to what has been done for the plane strain problem and from the definition of the exploded singularity presented in Figure 7, two regions are identified: (i) an inner one, corresponding to the sphere of radius $a$ and which is not actually visible in the original representation of the centre of dilation, and (ii) an outer one, consisting of the infinite porous medium complementary to the sphere. This definition also preserve the two characteristic parameters $r^{*}=a$ and $t^{*}=a^{2} / 4 c$.

Finally, the solution (18) is again decomposed in two components $u_{i}^{0}$ and $\Delta u_{i}$, corresponding, respectively, to the undrained response of the medium and the time-dependent component associated with the diffusive process. 


\subsubsection{Instantaneous Response}

As it has already been emphasized, the instantaneous response of the medium is governed by Navier equation with undrained material parameters and can, therefore, be obtained from the elastic solution by substituting the Poisson's ratio $\nu$ with the undrained one $\nu_{u}$. Using the influence functions for a circular dislocation loop originally derived by Paynter et al. (2007) in terms of Papkovich-Neuber displacement potentials and then corrected by Gordeliy and Detournay (2011), the instantaneous displacement field due to the insertion of the RD-triad is given by

$$
u_{i}^{0}=\frac{V}{3 \pi a^{2}}\left(u_{i 1}^{r c, 0}+u_{i 2}^{r c, 0}+u_{i 3}^{r c, 0}\right)
$$

where $u_{i k}^{r c, 0}$ is the elastic solution for a ring dislocation of radius $a$ characterized by a Burger vector parallel to the $x_{k}$-axis. This solution being expressed in terms of the generalized Lipschitz-Hankel integrals $P_{m, n, p}$ is not conveniently manipulable and will not be explicitly given here.

Similarly to what has been done for the plane strain configuration, one can identify a finite volume of solid $\Delta V_{c}^{0}$, which is instantaneously compressed in the inner region by considering the volumetric strain $\epsilon^{0}$, see Figure 9. Indeed, switching to spherical coordinates $(r, \theta, \phi)$ and considering the inner asymptotic expansion of $\epsilon^{0}$, we have

$$
\epsilon^{0}=-\frac{V\left(1-2 \nu_{u}\right)}{2 \pi a^{3}\left(1-\nu_{u}\right)}+\sum_{k=1}^{n-1} \rho^{2 k} \mathcal{F}_{k}(\theta, \phi)+\mathcal{O}\left(\rho^{2 n}\right)
$$

where $\rho=r / a \ll 1$ and the coefficients $\mathcal{F}_{k}$ are functions of the azimuthal and zenith angles $\theta=$ $\arctan \left(x_{2} / x_{1}\right)$ and $\phi=\arccos \left(x_{3} / r\right)$, only. It actually appears that the symmetry of the problem guarantee these coefficients to be such that

$$
\int_{0}^{\pi} \int_{0}^{2 \pi} \mathcal{F}_{k} \sin \phi \mathrm{d} \theta \mathrm{d} \phi=0
$$

for all $k$. The volume of solid instantaneously compressed in the inner region due to the insertion of the RD-triad can, therefore, be obtained by expanding the series to infinity and integrating over the sphere of radius $a$

$$
\begin{aligned}
\Delta V_{c}^{0} & =-\int_{0}^{a} \int_{0}^{\pi} \int_{0}^{2 \pi} \epsilon^{0} a^{2} \sin \phi \mathrm{d} \theta \mathrm{d} \phi \mathrm{d} r \\
& =\frac{2\left(1-2 \nu_{u}\right)}{3\left(1-\nu_{u}\right)} V
\end{aligned}
$$

The complement to this volume $\Delta V_{s}^{0}=V-\Delta V_{c}^{0}$, which represents the volume of solid displaced by the singularity in the outer outer region, is again smaller than $V$ meaning that only a fraction of the singularity strength is perceived in the outer region. As already emphasized for the plane strain problem, since these results are independent of $a$, they remain valid when the RD-triad is shrunk to the center of dilation and, therefore, explain the appearance of Poisson ratio in the classic Dougall solution.

\subsubsection{Transient Response}

In plane strain, it was mentioned that the time-dependent part of the poroelastic dislocation segment is created by a pair of equal and opposite fluid dipoles. Similarly, it can be shown that the timedependent part of the poroelastic ring dislocation is created by a ring fluid dipole (Appendix B). Consequently, the transient part of the solution for the RD-triad results from the superposition of three, mutually orthogonal, rings fluid dipole characterized by an intensity equal to $2 \eta c D_{n}$, see Figure 8. The displacement field can, therefore, be expressed as

$$
u_{i}=u_{i}^{0}+2 \eta c D_{n}\left(u_{i 1}^{b c}+u_{i 2}^{b c}+u_{i 3}^{b c}\right)
$$




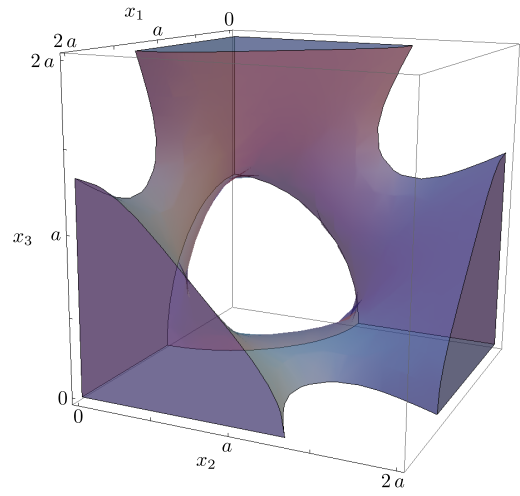

(a) Three-dimensional view of the region $\epsilon^{0}>0$

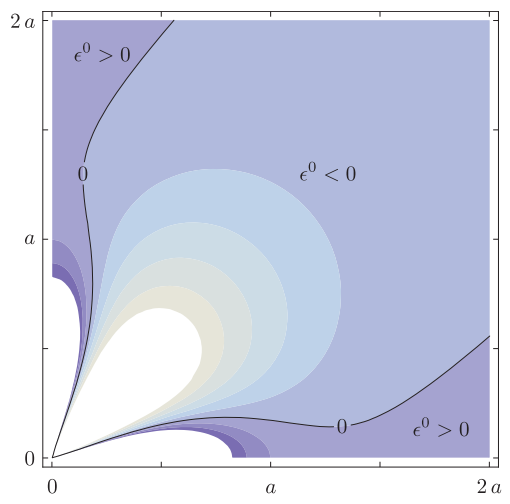

(d) Plane $x_{1}=a$

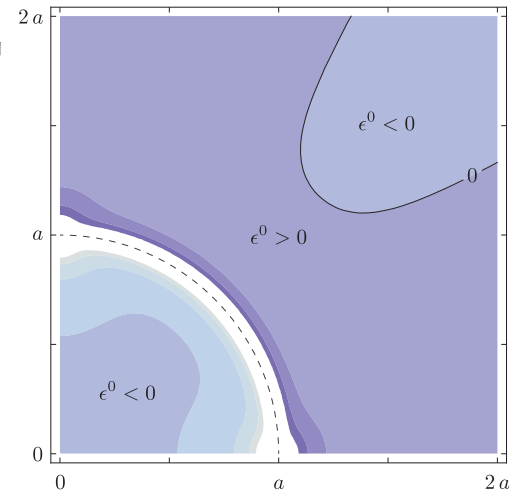

(b) Plane $x_{1}=0$

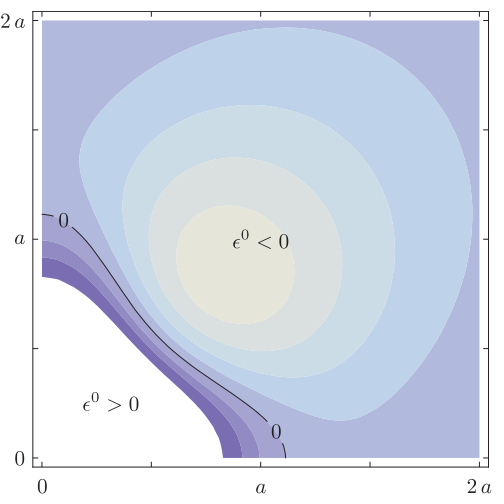

(e) Plane $x_{1}=3 a / 2$

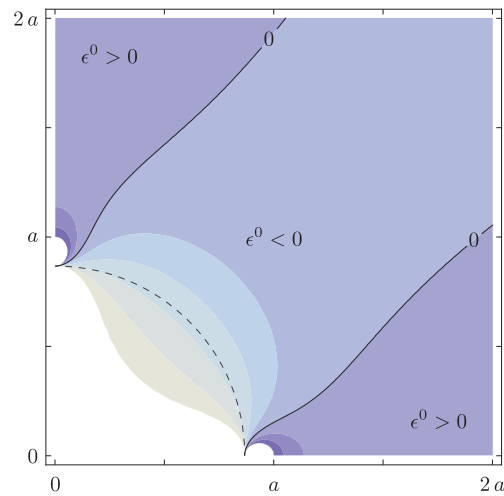

(c) Plane $x_{1}=a / 2$

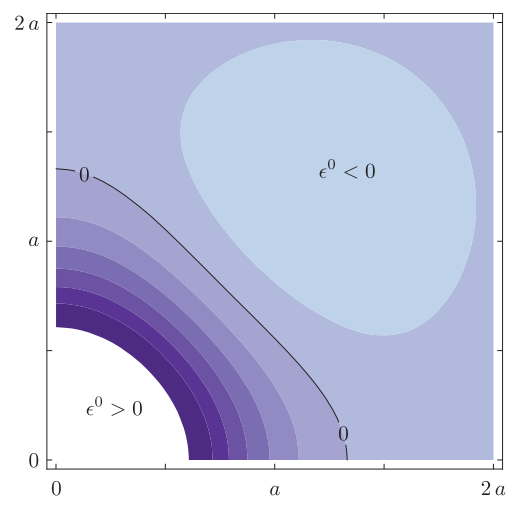

(f) Plane $x_{1}=2 a$

Figure 9: Instantaneous volumetric strain $\epsilon^{0}$. The solid is compressed inside the $\epsilon^{0}=0$ level curve (light blue), and is dilated outside.

where $u_{i}^{0}$ is the instantaneous part of the displacement, c.f. Eq. (19), and $u_{i k}^{b c}$ is the displacement due to a continuous ring fluid dipole of radius $a$ with normal in the $x_{k}$-direction.

The analogy with the plane strain problem is total, one can show that the volume $\Delta V_{c}$ of compressed solid increases progressively as interstitial fluid is expelled from the inner region, causing the volume $\Delta V_{s}$ of solid displaced in the outer region to decrease correspondingly. At large time, the excess of pore pressure diffuse and these two volumes of solid eventually attain

$$
\begin{aligned}
\Delta V_{c}^{\infty} & =\frac{2(1-2 \nu)}{3(1-\nu)} V \\
\Delta V_{s}^{\infty} & =\frac{1+\nu}{3(1-\nu)} V
\end{aligned}
$$

for $t \rightarrow \infty$. The variation of fluid content is again related to the volumetric strain by the relation $\zeta^{\infty}=\alpha \epsilon^{\infty}$ and the volume of fluid expelled from the inner region can be evaluated using Eq. (16) leading to $V_{f}^{\infty}=4 \eta V / 3$.

When the RD-triad is shrunk to the center of dilation, this volume of fluid appears to be instantaneously expelled from the inner region and, by continuity, injected in the outer one. It can actually be shown that this shrinking operation is, again, equivalent to the application of the Laplace operator 
to a continuous point fluid source (see Appendix (B)) such that

$$
\begin{aligned}
\zeta^{c c} & =\frac{2}{3} \eta c V \lim _{a \rightarrow 0} \frac{1}{\pi a^{2}}\left(\zeta_{1}^{b c}+\zeta_{2}^{b c}+\zeta_{3}^{b c}\right) \\
& =\frac{4}{3} \eta c V \zeta_{, k k}^{s c}
\end{aligned}
$$

and, therefore, leads to a relation similar to Eq. (17) with, in this configuration, $V_{f}=4 \eta V / 3$. For reasons similar to the one introduced in the previous section, one can conclude that the interstitial fluid thought to be injected in the medium originates from the point at which the center of dilation is introduced, the volume of fluid present in the porous solid remaining constant.

\section{Cylindrical \& Spherical Cavities}

In the previous sections, it has been emphasized that a fraction of the singularity strength is "lost" by compression of the inner region and that the volume of fluid seemingly injected in the medium is, in fact, expelled from this region due to its compression. The limiting process that was used to derive the solution for the center of dilation starting from Lamé solution for a circular or spherical cavity is, therefore, inadequate and one must slightly adjust it in order to account for this behavior.

As an alternative to the original limiting process, one can impose a displacement discontinuity of magnitude $D_{n}=V / 4 \pi a^{2}$ at the interface between the sphere of radius $a$ and the complementary infinite medium presented in Figure 10, with $a$ tending to zero. For the plane strain configuration, a similar process can be applied to the disk and the complementary infinite medium presented in Figure 11; the magnitude of the displacement discontinuity being $D_{n}=V / 2 \pi a$ in this configuration. For brevity and because the analogy between these two configurations is total, the details of this process are only presented for the three-dimensional configuration.

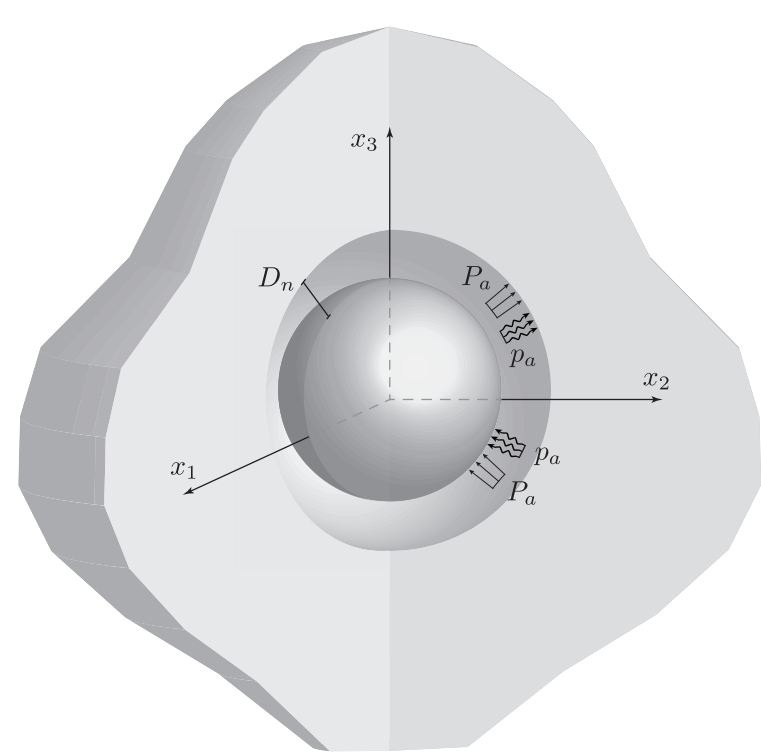

Figure 10: Spherical cavity and the embedded sphere (3D)

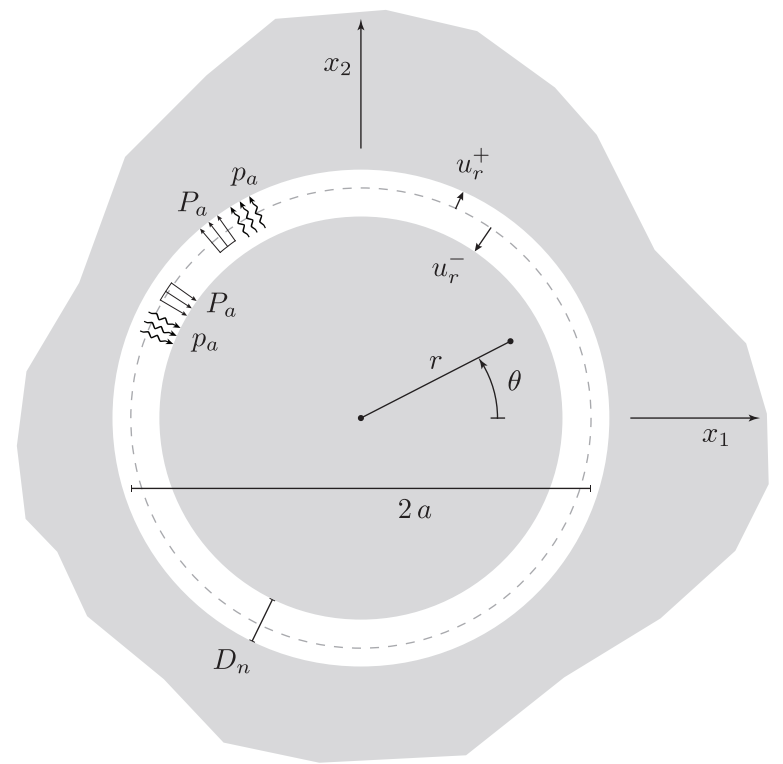

Figure 11: Cylindrical cavity and the embedded cylinder (plane strain)

The inner and outer problems, respectively associated with the sphere and the spherical cavity, have been considered individually and solved, in the Laplace domain, by superposition of two fundamental loading modes: (i) a step change in radial stress $\sigma_{r r}(a, t)=P_{a} H(t)$ while maintaining the pore 
pressure constant, and (ii) a step change in pore pressure $p(a, t)=p_{a} H(t)$ while maintaining constant radial stress. The details are presented in Appendix C. The solution associated with a constant displacement discontinuity is, therefore, build by superposition of the two loading modes and both radial stress $P_{a}$ and pore pressure $p_{a}$ are adjusted such that

$$
\tilde{u}_{r}^{e}(a, s)-\tilde{u}_{r}^{i}(a, s)=\frac{V s^{-1}}{4 \pi a^{2}}
$$

where the tilde stand for the Laplace transform while the superscripts $e$ and $i$ refer to the outer and inner solution, respectively. To close this problem, one must consider an additional condition; namely that interstitial fluid expelled from the inner sphere is injected in the complementary infinite medium through the surface of the spherical cavity

$$
\tilde{q}^{e}(a, s)-\tilde{q}^{i}(a, s)=0
$$

where the radial flux is derived from Darcy's law $\tilde{q}=-\kappa \tilde{p}_{, r}$ with the permeability $\kappa$.

Solving this system of two equations for the radial stress $P_{a}$ and the pore pressure $p_{a}$, the outer solution exhibits a zero volumetric strain at both short and large time which is in accordance with the classic Lamé solution. The time-dependence of the solution as well as its reliance on Poisson's ratios $\nu$ and $\nu_{u}$ are, however, representative of a diffusive process that arises from the compression of the sphere. This compression of the sphere, which is uniform at both short and long time

$$
\lim _{s \rightarrow 0 \text { or }+\infty} s \tilde{\epsilon}^{i}=-\frac{V}{\frac{4}{3} \pi a^{3}} \frac{2(1-2 \bar{\nu})}{3(1-\bar{\nu})}
$$

with $\bar{\nu}=\nu_{u}$ as $s \rightarrow \infty$ and $\bar{\nu}=\nu$ as $s \rightarrow 0$, eventually leads to the expulsion of a finite volume of fluid $V_{f}=4 \eta V / 3$.

As the sphere is shrunk to a point, i.e. its radius $a$ tends to zero, this volume of fluid appears to be instantaneously injected in the complementary infinite medium and the sphere acts as an instantaneous point fluid source. The compression of the sphere also explains the fact that only a fraction of the singularity strength is perceived in the outer region and, therefore, the dependence of the solution for the center of dilation on the Poisson's ratios $\nu$ and $\nu_{u}$. These conclusions are transposable to the plane strain configuration for which the volume of fluid expelled from the inner region is $V_{f}=\eta V$.

\section{Summary \& Conclusion}

In this paper, the elastic center of dilation and its poroelastic counterpart have been investigated in both three-dimensional and plane strain configurations. Comparison of the elastic nuclei of strain with the classic Lamé solutions for cylindrical and spherical cavities lead to detect an apparent inconsistent dependence on Poisson's ratio of Dougall's original solutions. In poroelasticity, the center of dilation was seen to be associated with the unexpected injection of a finite volume of fluid at the instant of loading.

To clarify these apparent paradoxes, two exploded views of this nucleus of strain have been considered and a compressed region was identified inside the singularity. As these alternative representations of the singularity are contracted to one point, the influence of the compressed zone on the outer domain remains and is revealed by the presence of Poisson's ratio in Dougall solution. This conclusion is in contradiction with Love's original intuition who stated that "The point must be in a cavity within the body [...]" (1920) which would result in a solution similar to the degenerated Lamé solution and, therefore, be independent of Poisson's ratio.

In poroelasticity, this compression has an additional effect on the solution. A finite volume of fluid is indeed expelled from the inner region and, by continuity, injected in the outer one. It is essential to understand that the volume of interstitial fluid present in the porous medium is not increased by the insertion of the center of dilation. 


\section{References}

Anderson, E. (1936). The dynamics of the formation of cone sheets, ring dykes and cauldron subsidences. Proceedings of the Royal Society of Edinburgh 56(206), 1972.

Biot, M. A. (1941). General theory of three-dimensional consolidation. Journal of Applied Physics 12(2), 155-164.

Boussinesq, J. (1885). Application des potentiels à l'étude de l'équilibre et du mouvement des solides élastiques. Gauthier-Villars.

Carvalho, J. L. and J. H. Curran (1998). Three-dimensional displacement discontinuity solutions for fluid-saturated porous media. International journal of solids and structures 35(34), 4887-4893.

Cheng, A. H.-D. and E. Detournay (1998). On singular integral equations and fundamental solutions of poroelasticity. International journal of solids and structures 35(34-35), 4521-4555.

Cleary, M. (1977). Fundamental solutions for a fluid-saturated porous solid. International Journal of Solids and Structures 13(9), 785-806.

Curran, J. H. and J. L. Carvalho (1987). A displacement discontinuity model for fluid-saturated porous media. In 6th ISRM Congress.

Detournay, E. and A. H.-D. Cheng (1987). Poroelastic solution of a plane strain point displacement discontinuity. Journal of Applied Mechanics 54(4), 783-787.

Detournay, E. and A. H.-D. Cheng (1993). Fundamentals of poroelasticity. In J. A. Hudson and C. Fairhurst (Eds.), Comprehensive Rock Engineering, Volume 2, Chapter 3, pp. 113-171. New York: Pergamon Press, Oxford, United Kingdom.

Dougall, J. (1897). A general method of solving the equations of elasticity. Proceedings of the Edinburgh Mathematical Society 16(1), 82-98.

Dundurs, J. (1969). Elastic interaction of dislocations with inhomogeneities. Mathematical theory of dislocations, $70-115$.

Eshelby, J. (1954). Distortion of a crystal by point imperfections. Journal of Applied Physics 25(2), $255-261$.

Eshelby, J. (1956). The continuum theory of lattice defects. Solid state physics 3, 79-144.

Geertsma, J. (1966). Problems of rock mechanics in petroleum production engineering. In $1^{\text {st }}$ ISRM Congress.

Geertsma, J. (1973a). A basic theory of subsidence due to reservoir compaction: the homogeneous case. Verhandelingen Kon. Ned. Geol. Mijnbouwk. Gen 28, 43-62.

Geertsma, J. (1973b). Land subsidence above compacting oil and gas reservoirs. Journal of Petroleum Technology 25(6), 734-744.

Gordeliy, E. and E. Detournay (2011). Displacement discontinuity method for modeling axisymmetric cracks in an elastic half-space. International Journal of Solids and Structures.

Groves, P. P. and D. J. Bacon (1969). Elastic centers of strain and dislocations. Journal of Applied Physics 40(10), 4207-4209. 
Gudmundsson, A. (1998). Formation and development of normal-fault calderas and the initiation of large explosive eruptions. Bulletin of volcanology 60(3), 160-170.

Kelvin, L. (1882). Note on the integration of the equations of equilibrium of an elastic solid. Cambridge and Dublin Mathematical Journal 3, 87-89.

Koehler, J. (1966). Elastic centers of strain and dislocations. Journal of Applied Physics 37(12), $4351-4357$.

Kröner, E. (1959). Allgemeine kontinuumstheorie der versetzungen und eigenspannungen. Archive for Rational Mechanics and Analysis 4, 273-334. 10.1007/BF00281393.

Lamé, G. (1852). Leçons sur la théorie mathématique de l'élasticité des corps solides. Bachelier.

Lisowski, M. (2006). Analytical volcano deformation source models. Volcano Deformation, 279-304.

Love, A. E. H. (1920). A Treatise on the Mathematical Theory of Elasticity. Cambrideg University Press.

McCann, G. and C. Wilts (1951). A mathematical analysis of the subsidence in the Long Beach - San Pedro area. California Institute of Technology.

Mogi, K. (1958). Relations between the eruptions of various volcanoes and the deformations of the ground surfaces around them. Bulletin of the Earthquake Research Institute, University of Tokyo 36, 99-134.

Olver, F. W. J., D. W. Lozier, and R. F. Boisvert (2010). NIST handbook of mathematical functions. Cambridge University Press.

Paynter, R., D. Hills, and A. Korsunsky (2007). The effect of path cut on somigliana ring dislocation elastic fields. International Journal of Solids and Structures 44(20), 6653-6677.

Rudnicki, J. (1981). On fundamental solutions for a fluid-saturated porous solid, by M. Cleary. Int. J. Solids Struct 17, 855-857.

Rudnicki, J. (1986). Fluid mass sources and point forces in linear elastic diffusive solids. Mechanics of Materials 5(4), 383-393.

Segall, P. (1989). Earthquakes triggered by fluid extraction. Geology 17(10), 942.

Segall, P. (1992). Induced stresses due to fluid extraction from axisymmetric reservoirs. Pure and Applied Geophysics 139(3), 535-560.

Segall, P. (2010). Earthquake and volcano deformation. Princeton University Press.

Segall, P., J. Grasso, and A. Mossop (1994). Poroelastic stressing and induced seismicity near the lacq gas field, southwestern france. Journal of geophysical research 99(B8), 15423-15.

\section{Appendix}

\section{A Poroelastic Singularities}

In this Appendix, some of the Green's functions presented by Cheng and Detournay (1998) for quasistatic isotropic poroelasticity are given. The following notations have been adopted

$$
r=|\boldsymbol{x}-\boldsymbol{\chi}|
$$




$$
\begin{gathered}
r_{, i}=\frac{\partial r}{\partial x_{i}}=\frac{x_{i}-\chi_{i}}{r} \\
\xi=\frac{r}{\sqrt{4 c t}}
\end{gathered}
$$

and use of erf and erfe, respectively, to denote the error function and the complementary error function, while $E_{1}(\eta)$ is the exponential integral (Olver et al., 2010)

$$
E_{1}(z)=\int_{z}^{\infty} \frac{e^{-\tau}}{\tau} \mathrm{d} \tau
$$

\section{A.1 Continuous Displacement Discontinuity}

$E_{i j k l}=-\frac{1}{2}\left(\delta_{i k} \delta_{j l}+\delta_{i l} \delta_{j k}\right) \delta(\boldsymbol{x}-\boldsymbol{\chi}) \mathrm{H}(t-\tau)$

$2 \mathrm{D}$

$$
\begin{aligned}
u_{i k l}^{d c}= & \frac{1}{4 \pi\left(1-\nu_{u}\right)} \frac{1}{r}\left[\left(1-2 \nu_{u}\right)\left(\delta_{k l} r_{, i}-\delta_{i l} r_{, k}-\delta_{i k} r_{, l}\right)-2 r_{, i} r_{, k} r_{, l}\right] \mathrm{H}(t-\tau) \\
& +\frac{\eta^{2} c}{2 \pi G \kappa} \frac{1}{r}\left[\left(4 r_{, i} r_{, k} r_{, l}-\delta_{k l} r_{, i}-\delta_{i l} r_{, k}-\delta_{i k} r_{, l}\right) \frac{1-e^{-\xi^{2}}}{\xi^{2}}+2\left(\delta_{k l} r_{, i}-r_{, i} r_{, k} r_{, l}\right) e^{-\xi^{2}}\right] \\
\zeta_{k l}^{d c}= & \frac{\eta}{\pi} \frac{1}{r^{2}}\left[2 r_{, k} r_{, l}-\delta_{k l}-2\left(\delta_{k l}-r_{, k} r_{, l}\right) \xi^{2}\right] e^{-\xi^{2}}
\end{aligned}
$$

$3 \mathrm{D}$

$$
\begin{aligned}
u_{i k l}^{d c}= & \frac{1}{8 \pi\left(1-\nu_{u}\right)} \frac{1}{r^{2}}\left[\left(1-2 \nu_{u}\right)\left(\delta_{k l} r_{, i}-\delta_{i l} r_{, k}-\delta_{i k} r_{, l}\right)-3 r_{, i} r_{, k} r_{, l}\right] \mathrm{H}(t-\tau) \\
& +\frac{\eta^{2} c}{8 \pi G \kappa} \frac{1}{r^{2}}\left\{4\left(\delta_{k l} r_{, i}-r_{, i} r_{, k} r_{, l}\right)\left[\operatorname{erfc}(\xi)+\frac{2}{\sqrt{\pi}} \xi e^{-\xi^{2}}\right]\right. \\
& \left.+\left(5 r_{, i} r_{, k} r_{, l}-\delta_{k l} r_{, i}-\delta_{i l} r_{, k}-\delta_{i k} r_{, l}\right)\left[\frac{3 \operatorname{erf}(\xi)}{\xi^{2}}-\frac{6}{\sqrt{\pi}} \frac{e^{-\xi^{2}}}{\xi}+2 \operatorname{erfc}(\xi)\right]\right\} \\
\zeta_{k l}^{d c}= & \frac{\eta}{2 \pi} \frac{1}{r^{3}}\left\{\left(3 r_{, k} r_{, l}-\delta_{k l}\right)\left[\operatorname{erfc}(\xi)+\frac{2}{\sqrt{\pi}} \xi e^{-\xi^{2}}\right]-\frac{4}{\sqrt{\pi}}\left(\delta_{k l}-r_{, k} r_{, l}\right) \xi^{2} e^{-\xi^{2}}\right\}
\end{aligned}
$$

\section{A.2 Instantaneous Fluid Source}

$$
\gamma=\delta(\boldsymbol{x}-\boldsymbol{\chi}) \delta(t-\tau)
$$

$2 \mathrm{D}$

$$
\begin{aligned}
u_{i}^{s i} & =\frac{\eta c}{2 \pi G \kappa} \frac{r_{, i}}{r}\left(1-e^{-\xi^{2}}\right) \\
\zeta^{s i} & =\frac{1}{\pi} \frac{1}{r^{2}} \xi^{2} e^{-\xi^{2}}
\end{aligned}
$$

$3 \mathrm{D}$

$$
\begin{aligned}
u_{i}^{s i} & =\frac{\eta c}{4 \pi G \kappa} \frac{r_{, i}}{r^{2}}\left[\operatorname{erf}(\xi)-\frac{2}{\sqrt{\pi}} \xi e^{-\xi^{2}}\right] \\
\zeta^{s i} & =\frac{1}{\pi^{3 / 2}} \frac{1}{r^{3}} \xi^{3} e^{-\xi^{2}}
\end{aligned}
$$




\title{
A.3 Continuous Fluid Dipole
}

\author{
$\gamma_{, k}=-\delta(\boldsymbol{x}-\boldsymbol{\chi})_{, k} \mathrm{H}(t-\tau)$
}

2D

$$
\begin{aligned}
& u_{i k}^{p c}=\frac{\eta}{8 \pi G \kappa}\left[\left(2 r_{, i} r_{, k}-\delta_{i k}\right) \frac{1-e^{-\xi^{2}}}{\xi^{2}}-\delta_{i k} E_{1}\left(\xi^{2}\right)\right] \\
& \zeta_{k}^{p c}=\frac{1}{2 \pi c} \frac{r_{, k}}{r} e^{-\xi^{2}}
\end{aligned}
$$

$3 \mathrm{D}$

$$
\begin{aligned}
& u_{i k}^{p c}=\frac{\eta}{16 \pi G \kappa} \frac{1}{r}\left\{\left(3 r_{, i} r_{, k}-\delta_{i k}\right)\left[\frac{\operatorname{erf}(\xi)}{\xi^{2}}-\frac{2}{\sqrt{\pi}} \frac{e^{-\xi^{2}}}{\xi}\right]+\left(2 r_{, i} r_{, k}-\delta_{i k}\right) \operatorname{erfc}(\xi)\right\} \\
& \zeta_{k}^{p c}=\frac{1}{4 \pi c} \frac{r_{, k}}{r^{2}}\left[\frac{2}{\sqrt{\pi}} \xi e^{-\xi^{2}}+\operatorname{erfc}(\xi)\right]
\end{aligned}
$$

\section{B Poroelastic Dislocation Loop}

In both elasticity and poroelasticity, the solution for a dislocation loop can be obtained by integration of the corresponding dislocation dipole over the region delimited by the loop. Let, for instance, consider the poroelastic climb dislocation corresponding to the planar region $\mathcal{D}$ characterized by $x_{3}=$ cst. and delimited by the curve $\partial \mathcal{D}$. If the displacement jump is constant and equal to $D_{n}$, one can express the solution as

$$
u_{i}=D_{n} \iint_{\mathcal{D}} u_{i 33}^{d c} \mathrm{~d} A
$$

where $u_{i k l}^{d c}$ is the displacement field due to the dislocation dipole $E_{i j k l}$, see Appendix A.1.

The instantaneous component of the solution, $u_{i}^{0}$, is given by the undrained part of the dislocation dipole and corresponds therefore to the elastic solution for the dislocation with undrained material parameters. The time-dependent part of the solution, $\Delta u_{i}$, incorporates the diffusion process and is given by the irrotational part of the dislocation dipole. At large time, i.e. as $t \rightarrow \infty$, the diffusion process dissipates and the elastic solution is recovered.

Plugging the definition (2) of the displacement discontinuity $E_{i j k l}$ in the diffusion equation (8) and identifying the second derivative of a delta function $\delta_{, k l}(\boldsymbol{x})$ with a point fluid quadrupole $\zeta_{, k l}^{s c}$, it can be shown that

$$
\zeta_{k l}^{d c}=2 \eta c\left(\zeta_{, k l}^{s c}-\delta_{k l} \zeta_{, j j}^{s c}\right)
$$

such that the variation of fluid content due to the climb dislocation under consideration is given by

$$
\zeta=-2 \eta c D_{n} \iint_{\mathcal{D}} \zeta_{, 11}^{s c}+\zeta_{, 22}^{s c} \mathrm{~d} A
$$

Using the divergence theorem, this surface integral can be converted to a contour integral

$$
\begin{aligned}
\zeta & =-2 \eta c D_{n} \int_{\partial \mathcal{D}} \boldsymbol{n} \cdot \nabla \zeta^{s c} \mathrm{~d} s \\
& =-2 \eta c D_{n} \int_{\partial \mathcal{D}} \frac{\partial \zeta^{s c}}{\partial n} \mathrm{~d} s
\end{aligned}
$$


where $\boldsymbol{n}=\left(n_{1}, n_{2}, 0\right)$ is the unit normal to the curve $\partial \mathcal{D}$. Similarly, the transient part of the dislocation dipole can be expressed in terms of point fluid quadrupoles such that

$$
\Delta u_{i}=-2 \eta c D_{n} \int_{\partial \mathcal{D}} \frac{\partial u^{s c}}{\partial n} \mathrm{~d} s
$$

The last two equalities (B.2) and (B.3), which remain valid for any planar region $\mathcal{D}$, express that transient part of a poroelastic climb dislocation is equivalent to a loop of continuous point fluid dipoles aligned with the normal $\boldsymbol{n}$ to the contour $\partial \mathcal{D}$ of the dislocation. Considering now these two equalities in the plane strain context, it is straightforward that the time-dependent part of the poroelastic dislocation segment is created by a pair of equal and opposite points fluid dipole of intensity $2 \eta c$.

\section{B.1 Ring Dislocation}

We are interested in the poroelastic solution for the climb dislocation corresponding to the inner disk cut $\varrho<a$ lying in the plane $x_{3}=0$ and characterized by the displacement jump

$$
u_{33}^{r c}\left(\varrho, 0^{+}\right)-u_{33}^{r c}\left(\varrho, 0^{-}\right)=D_{n} \mathrm{H}(a-\varrho)
$$

where $\varrho^{2}=x_{1}^{2}+x_{2}^{2}$ and $u_{i k}^{r c}$ is the displacement field due to a continuous ring dislocation of radius $a$ characterized by a Burger vector parallel to the $x_{k}$-axis.

Instantaneous Response Gordeliy and Detournay (2011) treated the elastic counterpart of this Volterra dislocation; one can therefore obtain the instantaneous component of the solution by substituting the Poisson's ratio $\nu$ with the undrained one $\nu_{u}$. The volumetric strain instantaneously induced by the opening of the ring dislocation is given by

$$
\epsilon_{3}^{r c, 0}=\frac{\left(1-2 \nu_{u}\right) D_{n}}{2 \pi\left(1-\nu_{u}\right)} \frac{\left(\varrho^{2}+x_{3}^{2}-a^{2}\right) E\left(\frac{4 a \varrho}{(a+\varrho)^{2}+x_{3}^{2}}\right)-\left[(a-\varrho)^{2}+x_{3}^{2}\right] K\left(\frac{4 a \varrho}{(a+\varrho)^{2}+x_{3}^{2}}\right)}{\left[(a-\varrho)^{2}+x_{3}^{2}\right] \sqrt{(a+\varrho)^{2}+x_{3}^{2}}}
$$

where $K(z)$ and $E(z)$ are the complete elliptic integrals of the first and second kind, respectively. At the instant of loading, the variation of fluid content is zero and the pore pressure therefore reduces to $p_{3}^{r c, 0}=-\alpha M \epsilon_{3}^{r c, 0}$ where $M$ is the Biot modulus (Detournay and Cheng, 1993).

Transient Response From Eq. (B.2), it appears that the variation of fluid content due to a ring dislocation is equivalent to the one induced by a ring fluid dipole of same radius. One can circumvent the apparent absence (to the authors knowledge) of an explicit solution for the continuous ring fluid dipole by considering its instantaneous counterpart and, then, use the fact that an instantaneous forcing function is the time derivative of its continuous homologue. Using the solution for the instantaneous point fluid source given in Appendix A.2 and following the integration path presented in Figure B.1 with $r^{2}=x_{3}^{2}+\varrho^{2}+a^{2}-2 a \varrho \cos \theta$, yields

$$
\zeta_{3}^{b i}=\frac{a e^{-\frac{a^{2}+\varrho^{2}+x_{3}^{2}}{4 c t}}}{16 \pi^{3 / 2}(c t)^{5 / 2}}\left[\varrho I_{1}\left(\frac{a \varrho}{2 c t}\right)-a I_{0}\left(\frac{a \varrho}{2 c t}\right)\right]
$$

where $I_{\nu}(z)$ is the modified Bessel function of the first kind of order $\nu$.

Integration of the former expression with respect to time yields the solution for the continuous ring fluid dipole such that

$$
\zeta_{3}^{r c}=2 \eta c D_{n} \int_{0}^{t} \zeta_{3}^{b i} \mathrm{~d} t
$$

which, to the authors knowledge, does not have a closed form. Since the pore pressure induced by the ring dislocation must eventually dissipates, it appears that the pore pressure due to the continuous 


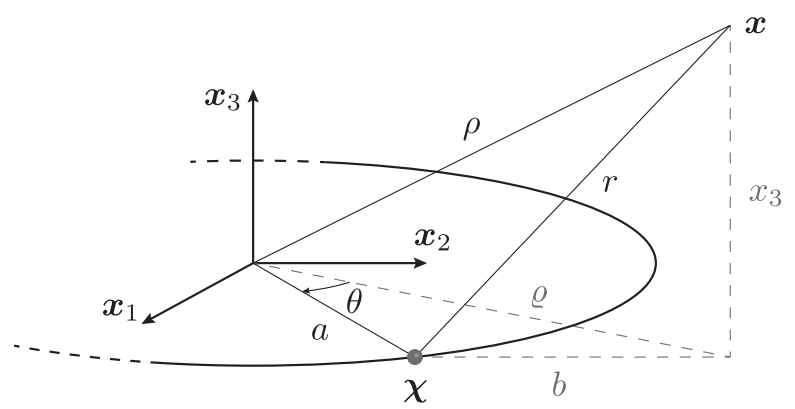

Figure B.1: Integration path for the ring fluid dipole

ring fluid dipole $p_{3}^{b c}=\kappa \zeta_{3}^{b c} / c$ tends to counterbalance $p_{3}^{r c, 0}$, the one instantaneously induced by the opening of the dislocation. The large time asymptotic of the ring fluid dipole is therefore given by

$$
\lim _{t \rightarrow \infty} \frac{\kappa}{c} \zeta_{3}^{r c}=\alpha M \epsilon_{3}^{r c, 0}
$$

where $\epsilon_{3}^{r c, 0}$ is the volumetric strain instantaneously induced by the opening of the ring dislocation, see Eq. B.4.

Considering now the limiting behavior, for $a$ tending to zero, of a ring dislocation associated with an opening $D_{n}=1 / \pi a^{2}$ inversely proportional to the dislocation area. From Eqs. (B.1) and (B.2), it appears that the ring fluid dipole tends to a set of two orthogonal point fluid quadrupoles

$$
\lim _{a \rightarrow 0} \frac{1}{\pi a^{2}} \zeta_{3}^{b c}=\zeta_{, 11}^{s c}+\zeta_{, 22}^{s c}
$$

Similar relations can be obtained for ring dislocation with Burger vectors parallel to the $x_{1}$ and $x_{2}$-axis such that

$$
\lim _{a \rightarrow 0} \frac{1}{\pi a^{2}}\left(\zeta_{1}^{b c}+\zeta_{2}^{b c}+\zeta_{3}^{b c}\right)=2 \zeta_{, k k}^{s c}
$$

\section{Cylindrical \& Spherical Cavities}

The approach presented here to solve the sphere and spherical cavity problems is similar to the one followed by Detournay and Cheng (1993) to solve the cylinder and borehole problems in plane strain. Both inner and outer problem are considered individually and solved, in the Laplace domain, by superposition of two fundamental loading modes: (i) a step change in radial stress $\sigma_{r r}(a, t)=P_{a} H(t)$ while maintaining the pore pressure constant, and (ii) a step change in pore pressure $p(a, t)=p_{a} H(t)$ while maintaining constant radial stress. The solution obtained by Detournay and Cheng in plane strain are also given in this appendix.

\section{C.1 Interior}

Due to the symmetry of the problem, the displacement field is irrotational and characterized by the only non-zero component $u_{r}(r, t)$. The pressure and radial stress, given by

$$
\begin{aligned}
p & =2 G \frac{\nu_{u}-\nu}{\alpha^{2}\left(1-2 \nu_{u}\right)(1-2 \nu)}(\zeta-\alpha \epsilon) \\
\sigma_{r r} & =2 G \varepsilon_{r r}+2 G \frac{\nu_{u}}{1-2 \nu_{u}} \epsilon-\alpha M \zeta
\end{aligned}
$$


respectively, are therefore functions of the the radius $r$, only. The volumetric strain reduces to

$$
\begin{aligned}
\epsilon & =\frac{\partial u_{r}}{\partial r}+2 \frac{u_{r}}{r} \\
& =\frac{1}{r^{2}} \frac{\partial}{\partial r}\left(r^{2} u_{r}\right)
\end{aligned}
$$

Moreover, if no fluid sources or body forces are present, the variation of fluid content satisfies the homogeneous diffusion equation

$$
\frac{\partial \zeta}{\partial t}-c \frac{1}{r^{2}} \frac{\partial}{\partial r}\left(r^{2} \frac{\partial \zeta}{\partial r}\right)=0
$$

and can be related to the volumetric strain through

$$
\frac{\partial \epsilon}{\partial r}=\gamma \frac{\partial \zeta}{\partial r}
$$

where the loading efficiency is defined as

$$
\gamma=\frac{\nu_{u}-\nu}{\alpha(1-2 \nu)\left(1-\nu_{u}\right)}
$$

Application of the Laplace transformation to the diffusion equation for $\zeta$ (to eliminate the time derivative operator) yields

$$
\frac{\mathrm{d}^{2} \tilde{\zeta}}{\mathrm{d} r^{2}}+\frac{2}{r} \frac{\mathrm{d} \tilde{\zeta}}{\mathrm{d} r}-\frac{s}{c} \tilde{\zeta}=0
$$

where the tilde denotes the Laplace transform

$$
\tilde{\zeta}(r, s)=\mathcal{L}[\zeta(r, t)]=\int_{0}^{\infty} \zeta(r, t) e^{-s t} \mathrm{~d} t
$$

and $s$ is the transform parameter. This differential equation has a general solution in terms of the modified spherical Bessel functions of the first and second kinds. Introducing the two dimensionless variables $\rho=r / a$ and $\beta=a \sqrt{s / c}$ where $a$ is the radius of the cavity, we have

$$
\tilde{\zeta}=C_{1} i_{0}(\varphi)+C_{2} k_{0}(\varphi)
$$

where $\varphi=\rho \beta, i_{0}(z)$ and $k_{0}(z)$ are the modified spherical Bessel functions of first and second kind, respectively, of order 0 (Olver et al., 2010). Due to the singular behavior of the functions $k_{\nu}(z)$ at the origin, the constant $C_{2}$ must be set to zero for the inner problem.

The volumetric strain can, therefore, be deduced by integration of Eq. (C.4)

$$
\tilde{\epsilon}=\gamma C_{1} i_{0}(\varphi)+C_{3}
$$

and the radial displacement is finally obtained by integration of Eq. (C.3)

$$
\tilde{u}_{r}=\frac{a \gamma}{\beta} C_{1} i_{1}(\varphi)+\frac{C_{3}}{3} r+\frac{C_{4}}{r^{2}}
$$

where $i_{1}(z)$ is the modified spherical Bessel functions of the first kind of order 1 . The constant $C_{4}$ must be set to zero so that the displacement field is bounded at the origin while the constants $C_{1}$ and $C_{3}$ are determined to satisfy the boundary conditions associated with the loading mode considered.

\section{C.1.1 Mode 1 Loading}

$\sigma_{r r}(a, t)=P_{a}$ and $p(a, t)=0$ 
$2 \mathrm{D}$

$$
\begin{aligned}
\frac{2 G}{a P_{a}} s u_{r}^{(1)} & =-\rho \frac{(1-\nu)\left(1-2 \nu_{u}\right) I_{0}(\beta)+2\left(\nu_{u}-\nu\right) \varphi^{-1} I_{1}(\varphi)}{(1-\nu) I_{0}(\beta)-2\left(\nu_{u}-\nu\right) \beta^{-1} I_{1}(\beta)} \\
\frac{s \tilde{p}^{(1)}}{p_{u}} & =\frac{(1-\nu)\left[I_{0}(\beta)-I_{0}(\varphi)\right]}{(1-\nu) I_{0}(\beta)-2\left(\nu_{u}-\nu\right) \beta^{-1} I_{1}(\beta)}
\end{aligned}
$$

where $p_{u}$ is the uniform increase of pore pressure at $t=0^{+}$

$$
\begin{aligned}
p_{u} & =p^{(1)}\left(r, 0^{+}\right) \\
& =\frac{2\left(\nu_{u}-\nu\right)}{\alpha(1-2 \nu)} P_{a}
\end{aligned}
$$

$3 \mathrm{D}$

$$
\begin{aligned}
\frac{2 G}{a P_{a}} s u_{r}^{(1)} & =-\rho \frac{(1-\nu)\left(1-2 \nu_{u}\right) i_{0}(\beta)+3\left(\nu_{u}-\nu\right) \varphi^{-1} i_{1}(\varphi)}{(1-\nu)\left(1+\nu_{u}\right) i_{0}(\beta)-6\left(\nu_{u}-\nu\right) \beta^{-1} i_{1}(\beta)} \\
\frac{s \tilde{p}^{(1)}}{p_{u}} & =\frac{(1-\nu)\left(1+\nu_{u}\right)\left[i_{0}(\beta)-i_{0}(\varphi)\right]}{(1-\nu)\left(1+\nu_{u}\right) i_{0}(\beta)-6\left(\nu_{u}-\nu\right) \beta^{-1} i_{1}(\beta)}
\end{aligned}
$$

where $p_{u}$ is the uniform increase of pore pressure at $t=0^{+}$

$$
\begin{aligned}
p_{u} & =p^{(1)}\left(r, 0^{+}\right) \\
& =\frac{3\left(\nu_{u}-\nu\right)}{\alpha(1-2 \nu)\left(1+\nu_{u}\right)} P_{a}
\end{aligned}
$$

\section{C.1.2 Mode 2 Loading}

$\sigma_{r r}(a, t)=0$ and $p(a, t)=p_{a}$

$2 \mathrm{D}$

$$
\begin{aligned}
\frac{2 G}{a p_{a}} s u_{r}^{(2)} & =\alpha(1-2 \nu) \rho \frac{\left(1-2 \nu_{u}\right) \beta^{-1} I_{1}(\beta)+\varphi^{-1} I_{1}(\varphi)}{(1-\nu) I_{0}(\beta)-2\left(\nu_{u}-\nu\right) \beta^{-1} I_{1}(\beta)} \\
\frac{s \tilde{p}^{(2)}}{p_{a}} & =\frac{(1-\nu) I_{0}(\varphi)-2\left(\nu_{u}-\nu\right) \beta^{-1} I_{1}(\beta)}{(1-\nu) I_{0}(\beta)-2\left(\nu_{u}-\nu\right) \beta^{-1} I_{1}(\beta)}
\end{aligned}
$$

$3 \mathrm{D}$

$$
\begin{aligned}
\frac{2 G}{a p_{a}} s u_{r}^{(2)} & =\alpha(1-2 \nu) \rho \frac{2\left(1-2 \nu_{u}\right) \beta^{-1} i_{1}(\beta)+\left(1+\nu_{u}\right) \varphi^{-1} i_{1}(\varphi)}{(1-\nu)\left(1+\nu_{u}\right) i_{0}(\beta)-6\left(\nu_{u}-\nu\right) \beta^{-1} i_{1}(\beta)} \\
\frac{s \tilde{p}^{(2)}}{p_{a}} & =\frac{(1-\nu)\left(1+\nu_{u}\right) i_{0}(\varphi)-6\left(\nu_{u}-\nu\right) \beta^{-1} i_{1}(\beta)}{(1-\nu)\left(1+\nu_{u}\right) i_{0}(\beta)-6\left(\nu_{u}-\nu\right) \beta^{-1} i_{1}(\beta)}
\end{aligned}
$$

\section{C.2 Exterior}

Because of the vanishing stress condition at infinity, the pore pressure uncouples from the stresses and, therefore, satisfies a homogeneous diffusion equation. The Laplace transform of this equation is similar to the diffusion equation for variation of fluid content Eq. C.5

$$
\frac{\mathrm{d}^{2} \tilde{p}}{\mathrm{~d} r^{2}}+\frac{2}{r} \frac{\mathrm{d} \tilde{p}}{\mathrm{~d} r}-\frac{s}{c} \tilde{p}=0
$$


The general solution is, therefore, again expressed in terms of the modified spherical Bessel functions of the first and second kinds

$$
\tilde{p}=C_{1} i_{0}(\varphi)+C_{2} k_{0}(\varphi)
$$

where $\varphi=\rho \beta$. Due to the unbounded behavior of the function $i_{0}(\varphi)$ for large $\varphi$, the constant $C_{1}$ must be set to zero for the outer problem.

The volumetric strain can be deduced from the pore pressure field by integration of the relation

$$
\frac{\partial \epsilon}{\partial r}=\frac{\eta}{G} \frac{\partial p}{\partial r}
$$

which leads to

$$
\tilde{\epsilon}=\frac{\eta}{G} C_{2} k_{0}(\varphi)+C_{3}
$$

Finally, the radial displacement can be obtained by integration of Eq. C.3

$$
\tilde{u}_{r}=-\frac{a \eta}{\beta G} C_{2} k_{1}(\varphi)+\frac{C_{3}}{3} r+\frac{C_{4}}{r^{2}}
$$

where $k_{1}(z)$ is the modified spherical Bessel functions of the second kind of order 1 . The constant $C_{3}$ must be set to zero so that the displacement field is bounded at large radius while the constants $C_{2}$ and $C_{4}$ are determined to satisfy the boundary conditions associated with the loading mode considered.

\section{C.2.1 Mode 1 Loading}

$\sigma_{r r}(a, t)=P_{a}$ and $p(a, t)=0$

2D

$$
\begin{aligned}
\frac{2 G}{a P_{a}} s \tilde{u}_{r}^{(1)} & =\frac{1}{\rho} \\
\frac{s \tilde{p}^{(1)}}{P_{a}} & =0
\end{aligned}
$$

$3 \mathrm{D}$

$$
\begin{aligned}
\frac{2 G}{a P_{a}} s \tilde{u}_{r}^{(1)} & =\frac{1}{2 \rho^{2}} \\
\frac{s \tilde{p}^{(1)}}{P_{a}} & =0
\end{aligned}
$$

This mode of loading corresponds to the classic Lamé solution in elasticity which is associated with a zero volumetric strain. Therefore, no diffusive process is generated and, consequently, the resulting solution is independent of time.

\section{C.2.2 Mode 2 Loading}

$\sigma_{r r}(a, t)=0$ and $p(a, t)=p_{a}$

\section{$2 \mathrm{D}$}

$$
\begin{aligned}
\frac{2 G}{a p_{a}} s \tilde{u}_{r}^{(2)} & =2 \eta\left[\frac{K_{1}(\beta)}{\varphi K_{0}(\beta)}-\frac{K_{1}(\varphi)}{\beta K_{0}(\beta)}\right] \\
\frac{s \tilde{p}^{(2)}}{p_{a}} & =\frac{K_{0}(\varphi)}{K_{0}(\beta)}
\end{aligned}
$$


3D

$$
\begin{aligned}
\frac{2 G}{a p_{a}} s \tilde{u}_{r}^{(2)} & =2 \eta\left[\frac{\beta k_{1}(\beta)}{\xi^{2} k_{0}(\beta)}-\frac{k_{1}(\varphi)}{\beta k_{0}(\beta)}\right] \\
\frac{s \tilde{p}^{(2)}}{p_{a}} & =\frac{k_{0}(\varphi)}{k_{0}(\beta)}
\end{aligned}
$$

